\title{
Improved Wall Shear Rate Method for Robust Measurements
}

\author{
S. Ricci ${ }^{1}$, A. Swillens ${ }^{2}$, A. Ramalli ${ }^{1}$, M.Cinthio ${ }^{3}$, P. Segers ${ }^{2}$, P. Tortoli ${ }^{1}$ \\ ${ }^{1}$ Department of Information Engineering, University of Florence, Italy \\ ${ }^{2}$ IBiTech-bioMMeda, Gent University, Belgium \\ ${ }^{3}$ Department of Biomedical Engineering, Lund University, Sweden \\ stefano.ricci@unifi.it
}

\begin{abstract}
The Wall Shear Rate (WSR) represents an important parameter correlated with cardiovascular diseases, like, for example the atherosclerotic plaque formation. The WSR can be obtained as the radial blood velocity gradient assessed in the wall proximity. The WSR is typically approximated by using flow models like Poiseuille and/or Womersley applied to the measured center-line velocity. However these models cannot account for the complex flow configurations generated by the real geometry of the vessel, and the WSR estimate is inaccurate. The direct measurement of the velocity gradient through a Doppler high-resolution multigate technique can achieve a better accuracy, but the signal near the wall is affected by clutter. In this work an improved velocity reconstruction method for WSR measurement is proposed. It is based on the measurement of the actual velocity profile and a two-step interpolation that reconstructs the velocity in the wall proximity. The method, verified through realistic multiphysics simulations of a carotid artery, achieves a 5\% RMS error for velocity reconstruction and a $\mathbf{- 1 0 . 5 \%}$ underestimation in WSR assessment. The method was also tested on 14 healthy volunteers.
\end{abstract}

Keywords- Wall Shear Rate; Multiphysics Simulations; Blood Velocity Profile.

\section{INTRODUCTION}

The Wall Shear Stress (WSS) is a parameter accounting for the frictional force that the blood exerts on the inner layer of the arterial wall. Several studies show that WSS directly influences the vessel endothelial functions and highlight a relation between the WSS and important cardiovascular diseases [1], including the atherosclerotic plaque progression.

The WSS can be obtained multiplying the blood viscosity by the Wall Shear Rate (WSR), i.e. the velocity gradient in the vessel radial direction calculated in the proximity of the wall. Due to the difficulty of directly measuring the velocity gradient, the WSR is often approximated by assessing only the center-line velocity and the diameter of the vessel. These are employed to estimate the velocity gradient by applying a parabolic profile (Poiseuille) flow model [2]. A more complex approach exploits the Womersley flow model, which accounts for pulsatility [3]. These methods, however, do not account for the complexity of the real flow profile that is strongly altered

This work was supported by the Swedish Foundation for International Cooperation in Research and Higher Education (STINT) and by the European Union's Seventh Framework Programme (FP7/2007-2013) IMI/115006 (SUMMIT). by the irregular arterial geometry. For example, the slight curvature of the common carotid artery produces skewed flow profiles that can't be predicted by the aforementioned flow models [4][5]. Their application in WSR assessment often produces high inaccuracies.

A different approach was recently proposed to overcome these difficulties. In [6], the actual flow profile was directly measured through a high-resolution Doppler multigate approach, and then differentiated to obtain the WSR [7]. Unfortunately, the echo-signal in the $0.5-1 \mathrm{~mm}$ region near the wall, i.e. where the gradient should be actually calculated, is frequently masked by clutter, especially in systole, so the velocity profile needs to be restored.

In this work we propose an improved velocity reconstruction method for WSS measurement based on a twostep interpolation process. The method was first verified through realistic multiphysics simulations [8], then implemented in the ULA-OP research system [6] and further tested on the common carotid arteries of 14 healthy volunteers.
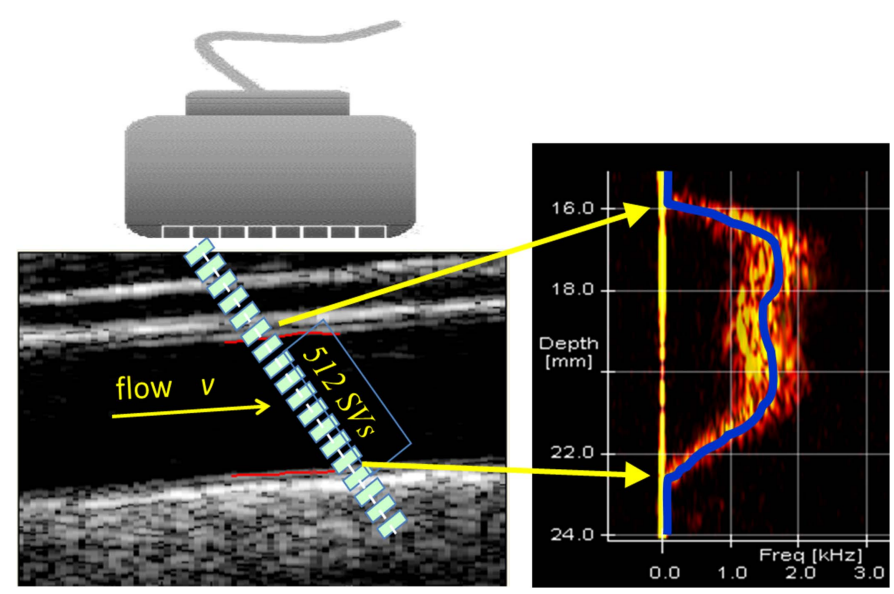

Fig. 1. A Doppler line with 512 SVs is placed across the vessel lumen (left). The spectra obtained from the SVs produce the spectral profile (right). The power-weighted mean frequency (right, blue line) is finally converted to velocity by the Doppler equation. 


\section{MATERIALS AND METHODS}

\section{A. Raw velocity profile extraction}

A pulse-wave (PW) Doppler line is placed across the lumen of the vessel of interest (see Fig.1, left). The fast-time data acquired from the investigation line are coherent-demodulated to produce the in-phase and quadrature (IQ) components, and subdivided in hundreds of small sample volumes (SVs). The slow-time I/Q data from each SV are processed through a weighted sliding-window 128-point FFT to produce the Doppler spectrum. The Doppler spectra obtained from each investigated SV at a given time, color coded and aligned in the rows of a matrix, represent the so-called spectral profile [6] (see Fig.1, right). The power-weighted mean frequency is calculated for each depth (see the blue line in Fig.1, right) and converted to velocity according to the Doppler equation.

\section{B. Velocity reconstruction and WSR measurement}

The small portion of the velocity profile near the walls is often unreliable due to the effect of the clutter. So it should be discarded and replaced by a 'reconstructed' profile. In this work we performed a two-step reconstruction. In the first step the two regions that extend for about $5-10 \%$ of the diameter from the wall positions towards the vessel lumen are located. The profile measured in these regions is substituted by a line that starts at the wall with velocity 0 and ramps up to join the remaining -measured-profile. In the second step, the resulting 3 -section curve is fitted by a polynomial interpolation of order 10. The WSR is finally selected as the maximum of the derivative of this fitting function, evaluated in the proximity of the proximal and distal walls.

\section{Multiphysics simulations}

The presented method aims at reconstructing the profile present in the complex flow configurations generated by pulsatility and non-regular geometries. Testing it by using the simple parabolic velocity profile would be not appropriate. Invitro or in-vivo tests can generate complex flow configurations, but the lack of a reliable ground-truth reference would hamper the assessment of the method accuracy. Multiphysics simulations, which integrate an ultrasonic simulator and advanced numerical methods to calculate complex flow

TABLE I. ULTRASOUND SIMULATION SET-UP

\begin{tabular}{lll}
\hline Parameter & Symbol & Value \\
\hline Center Frequency & fo & $7 \mathrm{MHz}$ \\
Speed of sound & $\mathrm{c}$ & $1500 \mathrm{~m} / \mathrm{s}$ \\
Element pitch & - & $0.245 \mathrm{~mm}$ \\
Total elements & - & 192 \\
Sampling Frequency & $\mathrm{f}_{\mathrm{c}}$ & $50 \mathrm{MHz}$ \\
Transmission cycles & - & $5 \mathrm{with}$ Hann Window \\
Focal ratio & $\mathrm{F} \#$ & 2.5 \\
Apodization window & - & Tukey \\
Pulse Repetition Frequency & $1 / \mathrm{T}_{\mathrm{PRI}}$ & $8 \mathrm{kHz}$ \\
Doppler angle & $\delta$ & $60^{\circ}$ \\
Packet size & - & 128 \\
Tx focus & - & $18 \mathrm{~mm}$ \\
Rx focus & - & Dynamic \\
\hline
\end{tabular}

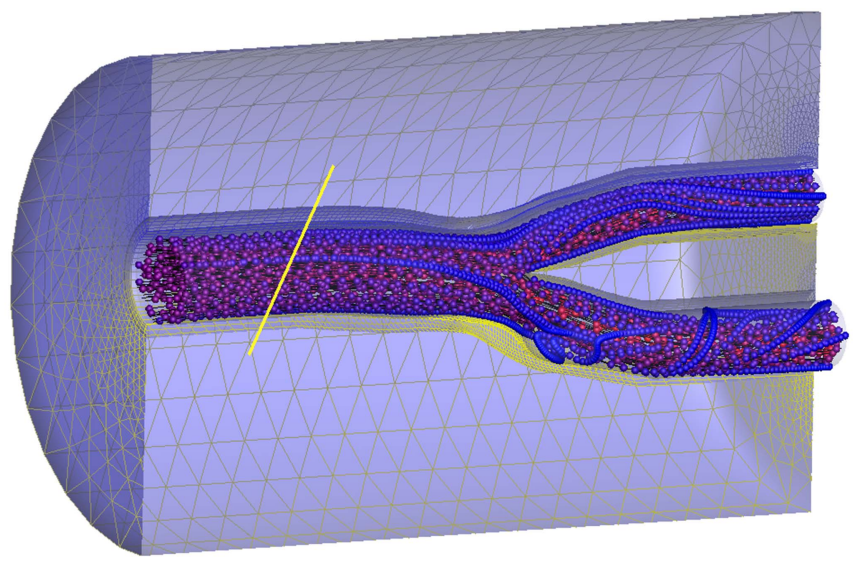

Fig. 2. Carotid artery bifurcation model used in simulations. The yellow segment represents the position of the Doppler line.

patterns and mechanical deformations [8], can be the solution to this problem.

The proposed method was thus validated with multiphysics simulations based on the geometry of a carotid artery bifurcation acquired through MRI from a volunteer (see Fig. 2). The carotid was filled with thousands of particles at random positions on which the ultrasound waves reflect. At every simulation step $(5 \mathrm{~ms})$ their positions were updated according to the spatially and temporally interpolated velocity fields obtained from computational fluid dynamic (CFD) calculations. A realistic pulsatile flow, measured from the common carotid artery of a healthy volunteer, was used for the boundary conditions. It featured $70 \mathrm{~cm} / \mathrm{s}$ peak velocity at systole and $13 \mathrm{~cm} / \mathrm{s}$ at diastole. Outflow percentages were imposed at the outlets $(35 \%$ at the external and $65 \%$ at the internal carotid). Blood was modeled as a Newtonian liquid with a viscosity of $3.5 \mathrm{mPa} \cdot \mathrm{s}$ and a density of $1050 \mathrm{~kg} / \mathrm{m}^{3}$. The walls and the tissue were modelled with different layers of scatterers. This allowed simulating the effect of the clutter as well. A Doppler line was placed across the lumen in the position reported by the yellow line visible in Fig. 2. FIELD II [9][10] was used for generating the radiofrequency data according to the probe and scan settings listed in Table I.

A cardiac cycle has been simulated. In particular the data in the temporal interval of systole and diastole have been processed as described in Sec. II.A and Sec. II.B. Finally, the velocity profile and the WSR obtained by applying the proposed method to simulated ultrasound data were compared to the ground-truth reference produced by the multiphysics calculations.

\section{In-vivo measurements}

The proposed method has been implemented on the research scanner ULA-OP [6]. ULA-OP is based on 5 Field Programmable Gate Arrays (FPGAs) that perform the most calculation intensive tasks like beamforming, and a Digital Signal Processor (DSP) in which 2 different software modules process simultaneously the Multigate Spectral Doppler and the B-mode. $1 \mathrm{~GB}$ of memory is available for saving data from all segments of the processing chain. ULA-OP is connected to a 

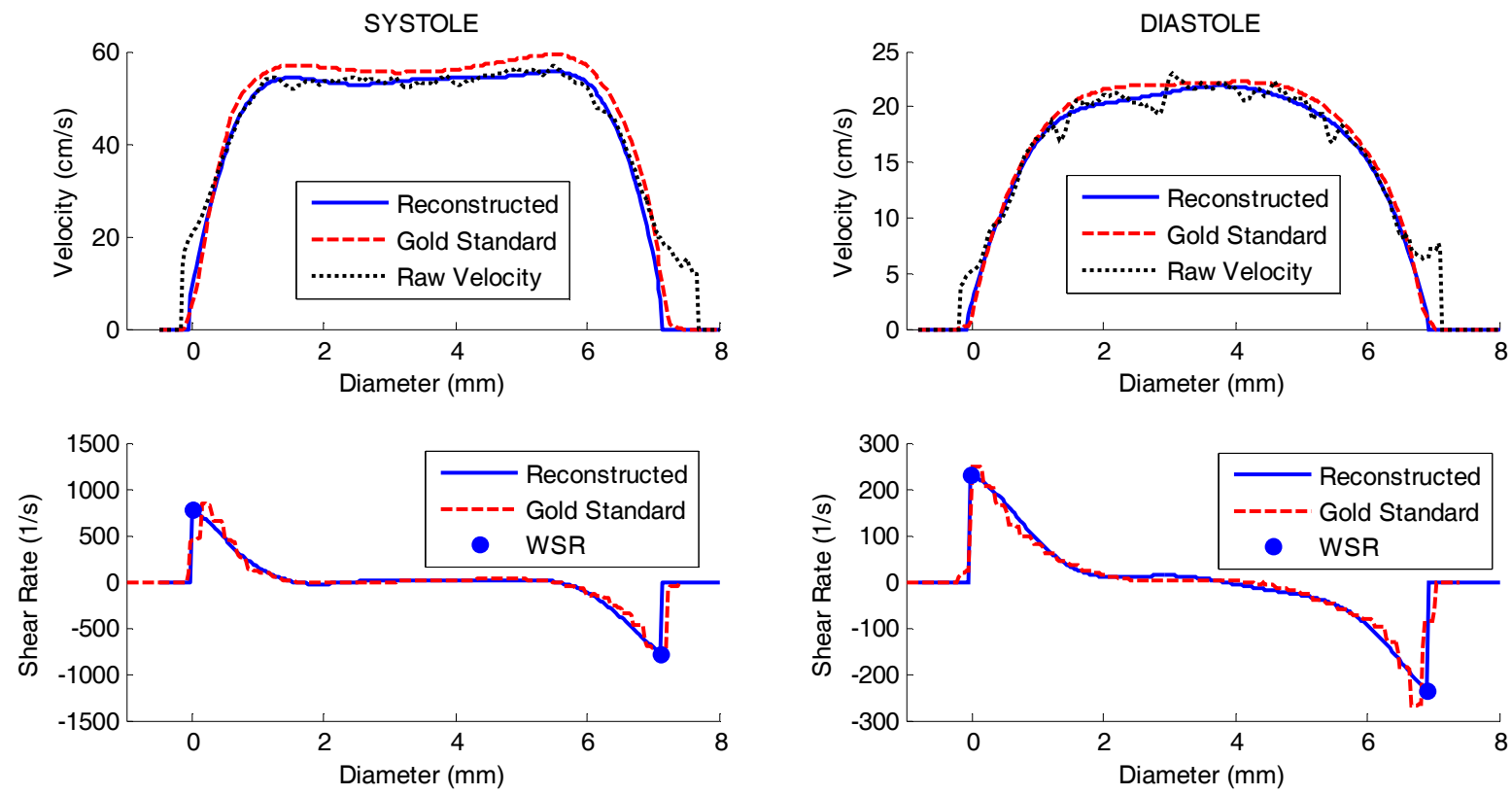

Fig. 3. Comparison between velocity (top) and shear rate (bottom) calculated by the proposed method (Reconstructed) and the reference from CFD simulation (Gold Standard) during the systole (left) and diastole (right) phases. The WSRs measured for the proximal and distal walls are highlighted by the filled circles.

PC where a specific software manages the acquisition parameters, displays in real-time the results and holds the acquired raw data.

In this experiment the ULA-OP was set in duplex-mode to obtain simultaneous B-mode images and PW Doppler data. ULA-OP was coupled to the LA523 probe (Esaote S.p.A., Florence, Italy) and programmed with the basic configuration reported in Table I. Some parameters, e.g. the transmission focus, pulse repetition frequency, Doppler angle, etc, were adjusted according to the specific conditions present during the volunteer investigation. During the exam, the B-mode and the spectral profile were elaborated and displayed in real-time so that the operator had an immediate feedback about the quality of acquired data.

This study was approved by the ethics committee of Lund University, Sweden, according to the Helsinki Declaration. Measurements were performed on the common carotid artery

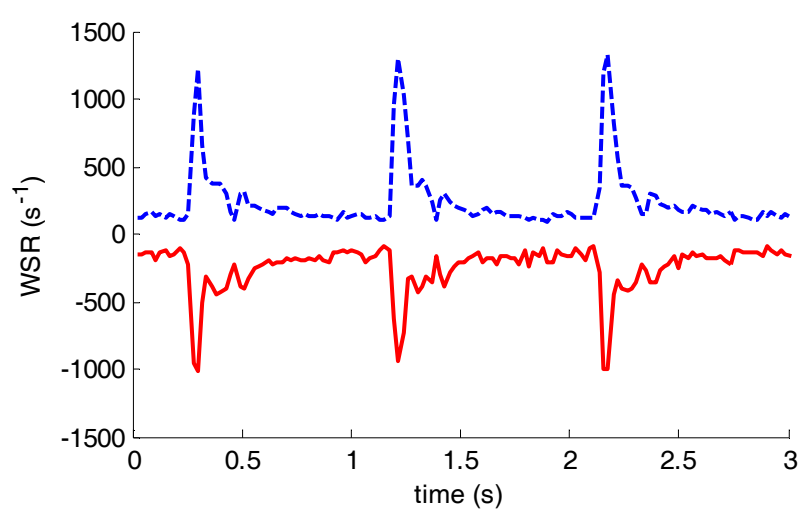

Fig. 4. WSR measured in the common carotid artery of a volunteer for proximal and distal walls (blue dashed and continuous red line, respectively)
(CCA) of 14 healthy normotensive volunteers, after informed consent was obtained. None of them reported previous cardiopulmonary diseases. During the measurements, the right CCA was scanned in the longitudinal direction and oriented horizontally in the B-mode image. A PW line crossing the lumen of the artery $2-3 \mathrm{~cm}$ proximal to the bifurcation, was steered to produce suitable spectral profiles. As soon as the correct positioning was achieved, B-mode and Doppler IQ data were saved over a time interval of about $3 \mathrm{~s}$.

The saved data were post-processed in MATLAB (The Mathworks, Natick, MA, USA). B-Mode images were recalculated and used for locating the wall positions through the ARTIC algorithm [11]. Wall positions were associated to the Doppler data, so that the WSR could be extracted from the proximal and distal walls as previously described.

\section{RESULTS}

\section{A. Multiphysics simulations}

Fig. 3 reports one example obtained by the multiphysics simulations. In particular the top row shows the velocity profiles present during systole (left) and diastole (right). Due to clutter, the velocity profile measured by ultrasound (dotted black curve labelled 'Raw velocity') is inaccurate at the proximal and distal wall regions (located at about 0 and $7 \mathrm{~mm}$, respectively). The velocity reconstructed by the proposed method is represented by the blue continuous curve (Reconstructed), which can be compared to the gold standard velocity predicted by CFD calculations (red dashed curve). The simulations showed that the velocity calculated from the Doppler ultrasound data, processed according to the proposed two-step interpolation method, is affected by a 5\% RMS error with respect to the gold standard. This result was calculated over several velocity profiles taken from the systolic and the diastolic regions, considered for their wall-to-wall extension. 


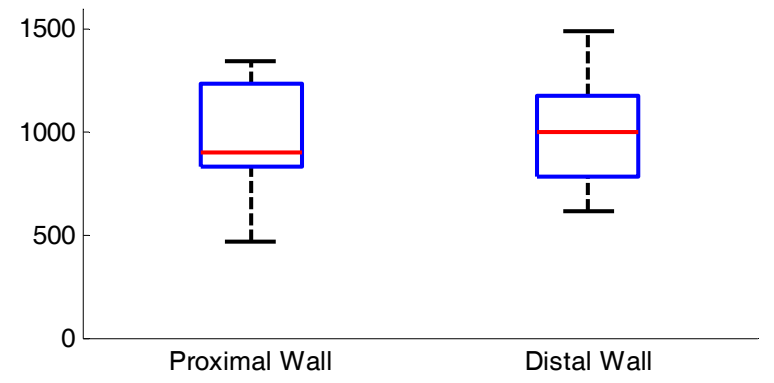

Fig. 5. Distribution of the WSRs measured in proximal (left) and distal (right) walls of 14 healthy volunteers. Distal WSRs are in absolute value. The box accounts for the $25^{\text {th }}$ and $75^{\text {th }}$ percentile, the internal horizontal line reports the median value, and the whiskers show the outmost samples.

The bottom row of Fig. 3 reports results of the shear rate estimations. The blue continuous curve (Reconstructed) and the red dashed curve (Gold standard) show the shear rates obtained by differentiating the corresponding velocity profiles. The measured WSRs, i.e. the maximum shear rate in the wall proximity, are represented by the filled circles. The WSR measurements showed an underestimation of $-10.5 \%$ with respect to the values calculated by CFD. This error was averaged over several frames taken in the systolic and diastolic regions.

\section{B. In-vivo measurements}

An example of WSR measurement obtained in-vivo is shown in Fig. 4. The WSR temporal trend measured near the proximal and distal walls are reported for 3 cardiac cycles. The WSR range measured on the 14 volunteers is reported in Fig. 5 for proximal (left) and distal (right) walls. The values are in the range $500-1400 \mathrm{~s}^{-1}$ and the median values are near $1000 \mathrm{~s}^{-1}$.

\section{DISCUSSION AND CONCLUSION}

In this work a method for robust WSR measurement was presented. The method assesses the velocity gradient by the direct measurement of the whole velocity profile through a multigate Doppler technique. The profile near the wall, distorted by the clutter, is effectively reconstructed by a twostep method (see Fig. 3). No hypothesis on particular vessel geometry or flow trend is required. A simulation environment integrating ultrasound and fluid-structure interaction (FSI) simulations, allowed the construction of synthetic ultrasound data of a physiologically realistic artery. These data were crucial to assess the method average accuracy that resulted 5\% (RMS) in velocity reconstruction and $-10.5 \%$ in WSR assessment.

The velocity gradient in the wall region is particularly high (see, e.g., Fig. 3 top row), so that any small source of error like, for example, misplaced wall position, signal noise, velocity uncertainty, can produce an important variation on the final WSR measurement. Nevertheless, the proposed method features a good reproducibility, like confirmed by the similarity of the WSR curves over the 3 different cardiac cycles visible in Fig. 4.
The Doppler method used in this work is affected by an uncertainty on Doppler angle measurement that directly affects the accuracy of the velocity estimation and thus the WSR measurement. However, the proposed method is compatible with vector Doppler techniques [12][13] that can help improving the attainable performances.

\section{REFERENCES}

[1] C.K. Zarins, D.P. Giddens, B.K. Bharadvaj, V.S. Sottiurai, R.F. Mabon, S. Glagov, "Carotid bifurcation atherosclerosis: quantitative correlation of plaque localization with flow velocity profiles and wall shear stress", Circ Res, 1983, vol 53, no.4, pp. 502-514, http://dx.doi.org/10.1161/ 01.RES.53.4.502

[2] C. Carallo, L.F. Lucca, M. Ciamei, S. Tucci, M.S. de Franceschi, "Wall shear stress is lower in the carotid artery responsible for a unilateral ischemic stroke", Atherosclerosis, 2006, vol. 185, no.1, pp. 108-113, http://dx.doi.org/10.1016/j.atherosclerosis.2005.05.019

[3] J.R. Blake, S. Meagher, K.H. Fraser, W.J. Easson, P.R. Hoskins, "A Method to Estimate Wall Shear Rate with a Clinical Ultrasound Scanner", Ultras. Med. \& Biol., 2008, vol. 34, no. 5, pp. 760-774, http://dx.doi.org/10.1016/j.ultrasmedbio.2007.11.003

[4] P. Tortoli, V. Michelassi, G. Bambi, F. Guidi, D. Righi, "Interaction between secondary velocities, flow pulsation and vessel morphology in the common carotid artery", Ultras. Med. \& Biol.,2003, vol.29, n.3, pp.407-415, http://dx.doi.org/10.1016/S0301-5629(02)00705-6

[5] J.P. Mynarda, B.A. Wassermanb, D.A. Steinman, "Errors in the estimation of wall shear stress by maximum Doppler velocity", Atherosclerosis, 2013 vol. 227, no. 2, pp. 259-266, http://dx.doi.org/10.1016/j.atherosclerosis.2013.01.026

[6] E. Boni, L. Bassi, A. Dallai, F. Guidi, A. Ramalli, S. Ricci, J. Housden, P. Tortoli, "A reconfigurable and programmable FPGA-based system for nonstandard ultrasound methods", IEEE Trans. Ultrason., Ferroelect., Freq. Contr., vol. 59 no. 7, pp. 1378 - 1385, 2012, http://dx.doi.org/10.1109/TUFFC.2012.2338

[7] P. Tortoli, T. Morganti, G. Bambi, C. Palombo, K.V. Ramnarine, "Noninvasive simultaneous assessment of wall shear rate and wall distension in carotid arteries", Ultras. Med. \& Biol., 2006, vol, 32, no. 11, pp. 1661-1670, http://dx.doi.org/10.1016/j.ultrasmedbio.2006.07.023

[8] A. Swillens, G. De Santis, J. Degroote, L. Lovstakken, J. Vierendeels, P. Segers, "Accuracy of carotid strain estimates from ultrasonic wall tracking: A study based on multiphysics simulations and in vivo data", IEEE Trans. Med.Imag., 2012, vol. 31, no. 1, pp. 131-139, http://dx.doi.org/10.1109/TMI.2011.2165959

[9] J. A. Jensen and N. B. Svendsen, "Calculation of pressure fields from arbitrarily shaped, apodized, and excited ultrasound transducers," IEEE Trans. Ultrason. Ferroelectr. Freq. Contr., vol. 39 no. 2, pp. 262-267, 1992, http://dx.doi.org/10.1109/58.139123.

[10] J.A. Jensen: "Field: A Program for Simulating Ultrasound Systems", Medical \& Biological Engineering \& Computing, vol. 34, no. 1, pp. 351-3531996.

[11] T. Nilsson, S. Segstedt, A.R. Ahlgren, S. Ricci, G. Ostling, P. Tortoli, J. Nilsson, T. Jansson, H.W. Persson, M. Cinthio, "A robust and fast method for arterial lumen diameter and intima-media thickness measurements", 2012 IEEE International Ultrasonics Symposium Proc., 2012, pp. 1678-1681, Dresden, http://dx.doi.org/10.1109/ULTSYM.2012.0421

[12] S. Ricci, L. Bassi, P. Tortoli, "Real Time Vector Velocity Imaging through Multigate Doppler and Plane Waves", IEEE Trans. Ultrason. Ferroelectr. Freq. Contr., Vol. 62, no. 2, pp. 314-324, 2014, http://dx.doi.org/10.1109/TUFFC.2014.2911

[13] I.K. Ekroll, A. Swillens, P. Segers, T.D.H. Torp, L. Lovstakken, "Simultaneous Quantification of Flow and Tissue Velocities Based on Multi-Angle Plane Wave Imaging", IEEE Trans. Ultrason. Ferroelectr. Freq. Contr., vol. 60 no. 4, pp. $727-738, \quad 2013$, http://dx.doi.org/10.1109/TUFFC.2013.2621 